\title{
Le ligand de RET est également impliqué dans la maladie de Hirschsprung
}

Les progrès constants de la génétique moléculaire permettent désormais d'envisager une approche mécanistique des maladies ou malformations réputées multigéniques. Suivant ce modèle, le phénotype dépend de l'action conjuguée de plusieurs locus, un gène dominant pouvant voir sa pénétrance modulée par un ou plusieurs gènes modificateur(s), ou un phénotype pouvant apparaître avec un seuil déterminé par l'effet additif de plusieurs allèles indépendants. La maladie de Hirschsprung pourrait être un modèle exemplaire dans ce domaine. Après la découverte de mutations du proto-oncogène RET dans la maladie de Hirschsprung en 1994 [1], puis du rôle surprenant de deux gènes participant à la voie de transmission du signal des endothélines, le récepteur EDNRB en $1995\left(\mathrm{~m} / \mathrm{s} n^{\circ} 8\right.$, vol. 11, p. 1172) [2], et son ligand l'endothéline 3 (EDN3) en 1996 [3], cette fin d'année est marquée par l'identification du ligand de Ret, le facteur neurotrophique GDNF $[4,5]$, et d'une molécule attachée à la membrane indispensable à l'action de ce ligand, GDNFR- $\alpha$ [6, 7]. Ces découvertes constituent des événements de première importance pour la compréhension des mécanismes moléculaires de la maladie de Hirschsprung. La maladie de Hirschsprung est une des plus fréquentes malformations du tube digestif ( 1 cas sur 5000 naissances). Elle est caractérisée par l'absence des cellules ganglionnaires du système nerveux entérique dans la partie terminale de l'intestin. Il en résulte des graves troubles de la motricité intestinale se révélant en général dès la période néonatale par une 1414 par une constipation opiniâtre [8]. Le système nerveux entérique a pour origine les crêtes neurales, à partir desquelles les neuroblastes primitifs migrent le long du tractus digestif, dans le sens oral-aboral. Ainsi, l'aganglionose de la maladie de Hirschsprung résulte d'une anomalie de migration, de différenciation ou de survie de ces cellules au cours du développement embryonnaire. Alors que les cas de maladie de Hirschsprung sont sporadiques en grande majorité $(80 \%)$, l'existence de formes familiales atteste de facteurs génétiques. Les analyses de ségrégations concluent à un mode de transmission multifactorielle incluant un (ou plusieurs) gène (s) dominant(s) de faible pénétrance [9]. La présence chez certains patients d'anomalies cytogénétiques sur des chromosomes variés, la faible pénétrance des mutations de $R E T$, et la prédominance de garçons atteints (rapport garçons/filles $=3,5$ ) sont autant d'arguments en faveur de l'hétérogénéité génétique de la maladie de Hirschsprung (les mutations de plusieurs gènes pouvant conduire à la maladie de façon indépendante), et appuient aussi l'hypothèse d'un mode héréditaire multigénique ; des anomalies combinées de plusieurs gènes peuvent être en cause chez un même patient.

Des mutations dans le gène codant pour le ligand de RET: confinnation de l'hétérogénéité génétique de la maladie de Hirschsprung

Le GDNF (glial cell line-derived neurotrophic factor), est un membre éloigné de la superfamille du TGF- $\beta$, doué d'un rôle trophique pour les neurones dopaminergiques et les moto- neurones $[10,11]$. Plusieurs travaux récents ont conduit à émettre l'hypothèse que GDNF devait également intervenir dans la mise en place du système nerveux entérique. L'étude de l'expression de GDNF chez l'embryon montrant un taux d'ARN messager très élévé dans le tube digestif et le rein [12] en constituait déjà le premier argument. La deuxième observation est venue de l'invalidation ciblée du gène $G d n f$ chez la souris $G d n f^{\prime-}$ aboutissant à l'état homozygote à un phénotype très proche de celui des souris Ret $^{-1-}$ : aganglionose digestive et agénésie rénale [13-15]. La similitude des souris $G d n f^{/-}$et $\operatorname{Ret}^{-/-}$suggérait aussi un lien étroit entre ces deux protéines. Ne connaissant ni le récepteur de GDNF, ni le ligand de RET, il était donc tentant de penser que GDNF était le (ou l'un des) ligand(s) de ce récepteur tyrosine-kinase orphelin depuis 1988. La réponse a été donnée par deux types d'approches différentes ayant fait l'objet de quatre publications concomitantes dans les numéros de Nature et de Cell datés des 4 juillet et 28 Juin 1996 [4-7]. La recherche du récepteur de GDNF dans une banque d'expression a permis d'identifier un complexe ternaire composé du ligand (GDNF), $\mathrm{du}$ récepteur tyrosine-kinase transmembranaire (RET) et d'une molécule fixée à la surface externe de la membrane (GDNFR- $\alpha$ ), corécepteur indispensable à l'activation de RET par GDNF [6, 7] (figure 1). D'autres études portant sur différents modèles cellulaires in vitro montrent clairement l'activation spécifique de RET par GDNF [4, 5] (voir pour plus de détail la mise au point sur ce sujet dans ce même numéro p 1408). 


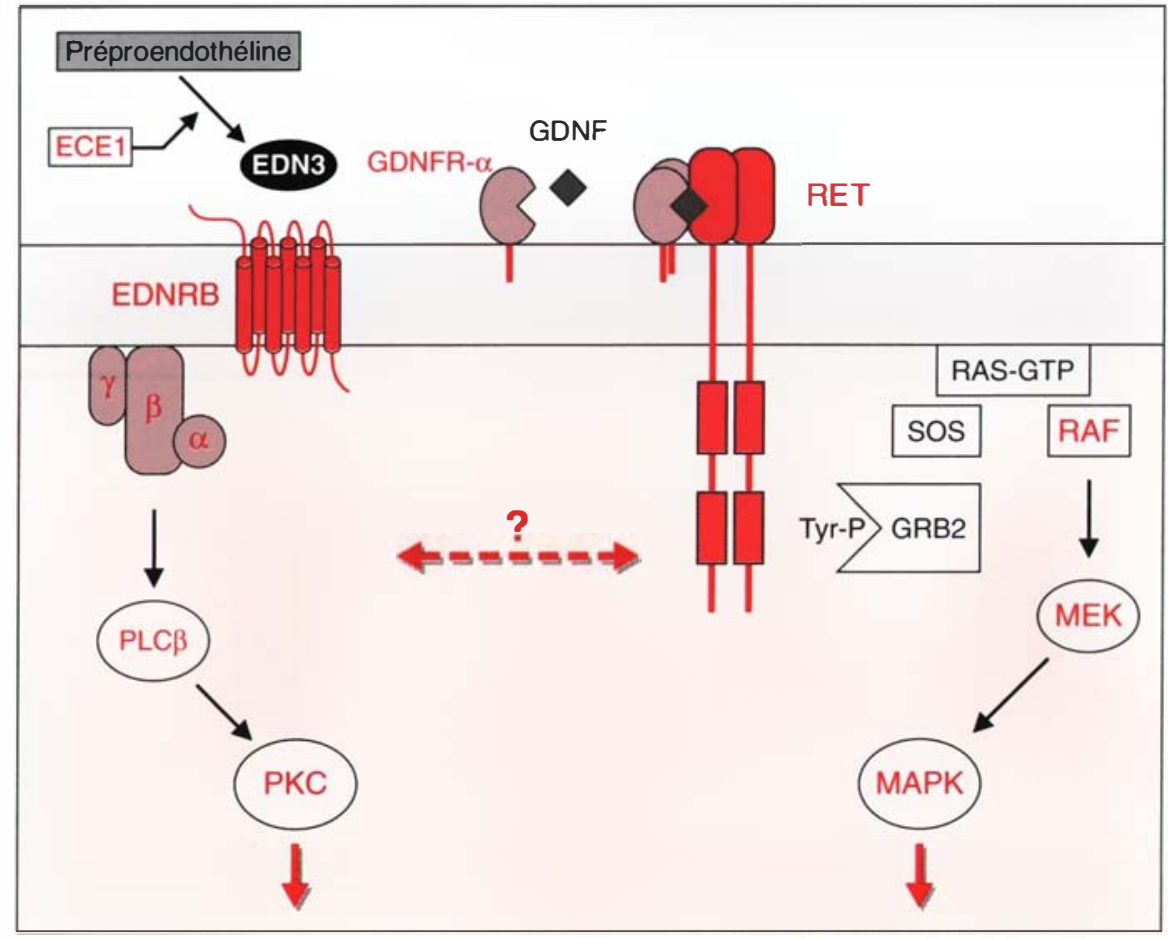

Figure 1. Gènes impliqués dans la maladie de Hirschsprung. Deux voies de transmission du signal sont concernées dans la maladie de Hirschsprung: la voie des endothélines avec des mutations d'un ligand (endothéline 3, EDN3), de son récepteur membranaire couplé aux protéines $G$ (EDNRB) et, vraisemblablement, d'une enzyme indispensable à la maturation de ce ligand (enzyme de conversion de l'endothéline, ECE-1). L'autre voie impliquée passe par le proto-oncogène RET, récepteur de type tyrosine-kinase, et son ligand GDNF. Le corécepteur GDNFR- $\alpha$, indispensable à l'activation de RET, ainsi que les autres protéines participant à la transmission du signal représentent donc de bon gènes candidats pour la maladie de Hirschsprung. $P L C \beta$ : phospholipase $C \beta, P K C$ : phosphokinase $C$; MAPK: mitogen activated protein kinase; MEK: MAPKJERK kinase.

Compte tenu du phénotype observé chez la souris $G d n f^{\prime-}$ et de la confirmation dans différents modèles de l'activation spécifique de RET par GDNF, il semblait très probable que ce facteur neurotrophique puisse également intervenir dans la mise en place du système nerveux entérique, et constituait donc un très bon gène candidat pour la maladie de Hirschsprung.

Le recherche de mutations dans la séquence codante de $G D N F$ dans une grande série de patients atteints de maladie de Hirschsprung (175 sujets atteints) s'est effectivement révélée fructueuse, avec l'identification de trois mutations faux-sens (P21S, R93W, D150N) situées dans des régions conservées chez le rat, à proximité d'un site de clivage du pro- trées ou vraisemblables du gène codant pour RET [16]. Ainsi, la mutation P21S a été retrouvée dans une famille multiplex chez deux individus atteints qui, seuls dans une fratrie de six, partageaient les mêmes haplotypes au locus $R E T$, indiquant qu'une mutation ou un variant non détectée de ce gène pourrait contribuer au phénotype chez ces deux enfants. Plus convaincante encore est l'association à la mutation R93W de GDNF (figure 2) d'une mutation silencieuse de l'exon 11 de RET (S649S) qui a pour effet de démasquer un site cryptique accepteur d'épissage. Il en résulte un variant de RET plus court de 20 acides aminés dans le domaine transmembranaire. L'existence dans une même famille et chez un même patient de mutations dans deux gènes différents suggère que la maladie de Hirschsprung pourrait résulter de l'effet combiné (ou additif) d'anomalies du ligand (GDNF) et de son récepteur (RET).

La situation est probablement encore plus complexe. En effet alors que le variant S649S de RET est présent chez tous les membres d'une grande famille, la mutation R93W de $G D N F$ n'a pas été retrouvée chez tous les individus atteints; en revanche, elle existe chez plusieurs individus sains. D'autres locus doivent donc intervenir pour influencer l'expression de $R E T$ et de GDNF. De fait, l'hypothèse du multigénisme pour la maladie de Hirschsprung a déjà été évoquée par A. Chakravarti à propos d'une grande famille d'origine mennonite dans laquelle une mutation faux-sens du gène $E D N R B$ (W276C) associée de façon non aléatoire à un polymorphisme du gène RET, suggérait l'interaction des voies de transmission du signal via les protéines G (EDNRB), d'une part, et via les récepteurs tyrosine-kinase (RET), d'autre part (figure 1) [17]. Cela évoque aussi l'éventualité dans la maladie de Hirschsprung d'autres gènes candidats codant pour des protéines participant également à ces deux voies de transmission des signaux cellulaires à partir de récepteurs membranaires.

La découverte d'anomalies de locus indépendants chez un même individu fait donc de la maladie de Hirschs- 


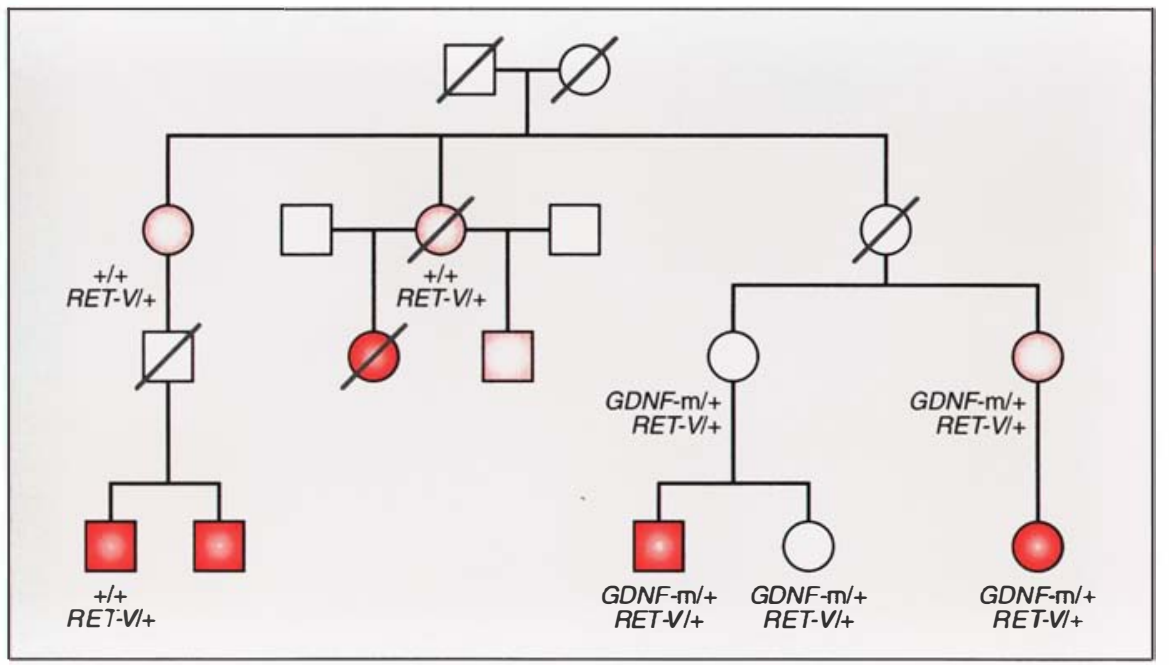

Figure 2. Pedigree d'une famille au sein de laquelle coségrègent une mutation de GDNF et un variant de RET. GDNF-m/+: mutation faux-sens (R93W) hétérozygote de GDNF. RET-V/+: variant de RET (S649S) démasquant un site cryptique d'épissage, ayant pour effet l'apparition d'une isoforme délétée de 20 acides aminés au niveau du domaine transmembranaire. Alors que le variant S649S de RET est présent chez tous les membres de cette famille, la mutation $R 93 \mathrm{~W}$ de GDNF n'a pas été retrouvée chez tous les individus atteints; en revanche, elle existe chez plusieurs individus sains. D'autres locus doivent donc intervenir pour influencer l'expression de RET et de GDNF.

prung un des tout premiers modèles de maladie humaine dans laquelle l'hypothèse du multigénisme pourrait être validée au niveau moléculaire. L'étude in vitro des effets combinés des mutations de ces différents gènes est désormais une étape indispensable pour confirmer cette analyse

\section{RÉFÉRENCES}

1. Edery P, Pelet A, Mulligan LM, Abel L, Attie $\mathrm{T}$, et al. Long segment and short segment familial Hirschsprung's disease: variable clinical expression at the $R E T$ locus. J Med Genet 1994; 31 : 602-6.

2. Puffenberger EG, Hosoda $\mathrm{K}$, Washington SS, Nakao K, de Wit D, Yanagisawa M, Chakravarti A. A missense mutation of the endothelin-B receptor gene in multigenic Hirschsprung's disease. Cell $1994 ; 79$ : 1257 66.

3. Edery P, Attie T, Amiel J, Pelet A, Eng C,
9. Bolande, R. The neurocristopathies: a unifyng concept of disease arising in neural crest maldevelopment. Hum Pathol 1993; 5 : 409-29.

10. Lin L, Doherty D, Lile I, Bektesh S. Collins, F. GDNF: a glial cell line-derived neurotrophic factor for midbrain dopaminergic neurons. Science 1993; 260 : 1130-2

11. Oppenheim RW, Houenou LJ, Johnson JE, Lin LF, Li L, Lo AC, Newsome AL, Prevette DM, Wang $S$. Developing motor neurons rescued from programmed and axotomy-induced cell death by GDNF. Nature $1995 ; 373$ : 344-6.

12. Trupp M, Ryden M, Jornvall H, Funakoshi $H$, Timmusk $T$, Arenas $E$, Ibanez CF. Peripheral expression and biological activities of GDNF, a new neurotrophic factor for avian and mamalian peripheral neurons. I Cell Biol 1995; 130 : 137-48.

13. Sanchez M, Silos-Santiago I, Frisen J, He B, Lira SA, Barbacid M. Renal agenesis and the absence of enteric neurons in mice lacking GDNF. Nature $1996 ; 382: 70-3$.

14. Pichel JG, Shen L, Sheng HZ, Granholm AC, Drago J, et al. Defects in enteric innervation and kidney development in mice lacking GDNF. Nature 1996; 382 : 73-6.

15. Moore MW, Klein RD, Fariñas I, Sauer H, Armanini M, Phillips H, Reichardt LF Ryan AM, Carven-Moore K, Rosenthal A. Renal and neuronal abnormalities in mice lacking GDNF. Nature 1996; 382: 76-9.

16. Salomon R, Attié T, Pelet A, Bidaud C, Eng C, Amiel J, Sarnacki S, Goulet $O$, Ricour C, Nihoul-Fékété C, Munnich A, Lyonnet $\mathrm{S}$. Germline mutations of the RET ligand, GDNF, are not sufficient to cause Hirschsprung disease. Nature Genet 1996; 14: 345-7.

A, Lyonnet S. Mutation of the endothelin-3 gene in the Waardenburg-Hirschsprung disease (Shah-Waardenburg syndrome) Nature Genet $1996 ; 12$ : 442-4.

4. Durbec P, Marcos-Guttierez CV, Kilkenn G, Grigoriou M, Wartiowaara K, et al. GDNF signalling through the Ret receptor tyrosine kinase. Nature 1996; 381 : 789-93.

5. Trupp M, Arenas E, Fainsilber M, Nilsson AS, Sieber BE, et al. Functional receptor for GDNF encoded by the c-ret proto-oncogene. Nature $1996 ; 381$ : 785-9.

6. Treanor J, Goodman L, de Sauvage $\mathbf{F}$, Stone DM, Poulsen KT, et al. Characterization of a multicomponent receptor for GDNF. Nature 1996; 382: 80-3.

7. Jing S, Wen D, Yu Y, Holst PJ, Luo Y, et al. GDNF-induced activation of the Ret protein tyrosine kinase is mediated by GDNFR- $\alpha$, a novel receptor for GDNF. Cell 1996; 85: 1113-24.

8. Rescorla F, Morrison A, Engles D, West K, Grosfeld J. Hirshsprung's disease. Evaluation of mortality and long-term function in 260 cases. Arch Surg 1992; 127: 934-42.
17. Chakravarti A. Endothelin receptormediated signaling in Hirschsprung disease. Hum Molecul Genet 1996; 5: 303-7.

\section{Rémi Salomon \\ Tania Attié \\ Chrystelle Bidaud \\ Stanislas Lyonnet \\ Amold Munnich}

Inserm U.12, hôpital Necker, 149, rue de Sèrres, 75743 Paris Cedex 15, France.

\section{TIRÉS À PART}

S. Lyonnet. 